\title{
Oscillations in g-mode period spacings in red giants as a way to determine their state of evolution
}

\author{
M. S. Cunha, ${ }^{1,2}$, D. Stello, ${ }^{3,4}$, P.P. Avelino, ${ }^{1,2}$, and J. Christensen-Dalsgaard, ${ }^{4}$
}

1 Instituto de Astrofísica e Ciências do Espaço, Universidade do Porto, CAUP, Rua das Estrelas, 4150762 Porto, Portugal

2 Centro de Astrofísica e Faculdade de Ciências, Universidade do Porto, Rua das Estrelas, 4150-762 Porto, Portugal

3 Sydney Institute for Astronomy (SIfA), School of Physics, University of Sydney, NSW 2006, Australia

4 Stellar Astrophysics Centre, Department of Physics and Astronomy, Aarhus University, Ny Munkegade 120, DK-8000 Aarhus C, Denmark

\begin{abstract}
In this work we consider the sensitivity of gravity-mode period spacings to sharp changes in the inner structure of red giant stars, more specifically in the buoyancy frequency inside the g-mode propagation cavity. Based on a comparison between the solutions to the linear pulsation equations in the Cowling approximation for pure g-modes with results obtained with a full oscillation code we identify and correctly interpret the signature of the above-mentioned sharp variations in the period spacings. Two examples, of red giant models in different evolutionary phases, are discussed. Detection of these signatures in CoRoT, Kepler or future PLATO red-giant stars would pin down their evolutionary state in an unprecedented way.
\end{abstract}

\section{Introduction}

Red giant stars exhibit oscillations of mixed character, characterized by having the properties of an internal gravity wave (g-mode) in the deeper layers of the star, while maintaining the properties of an acoustic wave (p-mode) in the stellar envelope. The degree of mixing depends crucially on the frequency of the wave. In many cases, the waves are essentially trapped in the radiative part of the star's interior, behaving similarly to pure g-modes, but at frequencies close to the resonant acoustic frequencies (the frequencies p-modes would have in the absence of coupling with the g-modes) the modes acquire a stronger mixed character and have a significant part of their energy in the convective envelope.

Gravity waves propagate only where there is no convection and both their propagation cavity and periods depend on the buoyancy frequency. Sharp variations in the buoyancy frequency can shift the periods of g-modes away from their asymptotic values and, thus, leave a signature in the period spacings (defined as the difference between the periods of modes of the same degree and consecutive radial orders). Modes of mixed character will also be affected by this sharp variations, as they have the properties of a g-mode in the star's deepest layers.

In the following sections we will provide two examples of the effect that sharp variations in the buoyancy frequency may leave on the period spacings of red giant stars.

This is an Open Access article distributed under the terms of the Creative Commons Attribution License 4.0, which permits unrestricted use, distribution, and reproduction in any medium, provided the original work is properly cited. 


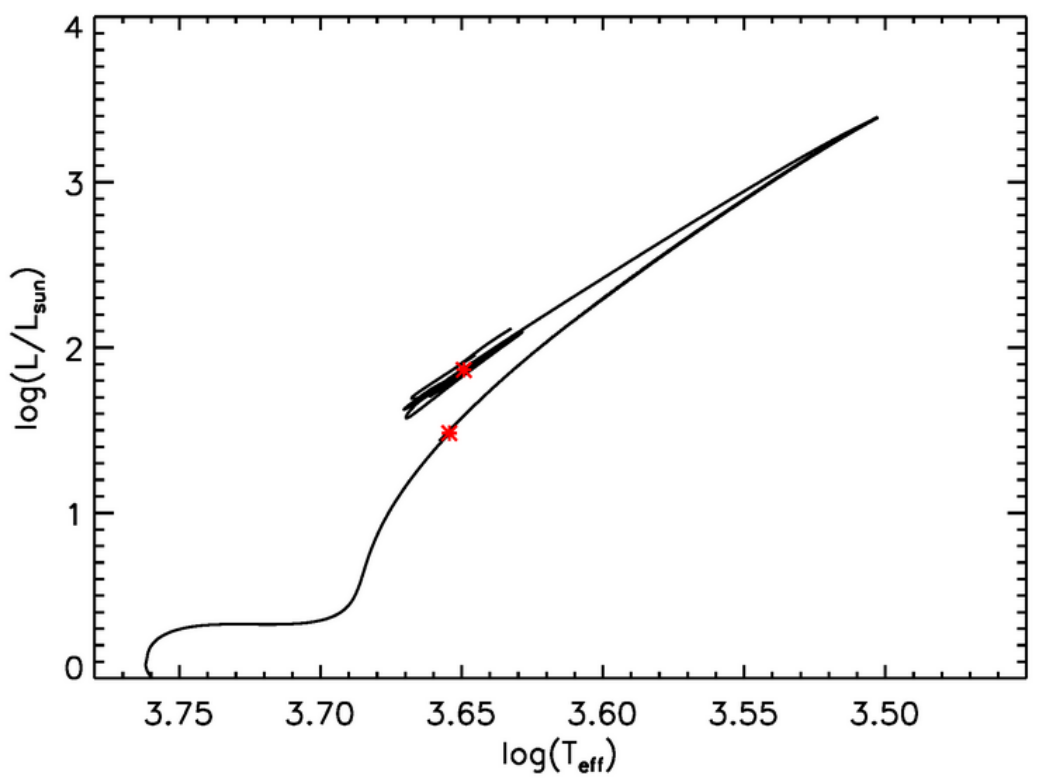

Fig. 1. HR diagram showing the evolutionary status of the two $1 \mathrm{M}_{\odot}$ red giant models considered in this work. The lower luminosity model is on the red giant branch and the higher luminosity model is between helium flashes. Evolutionary track computed with the evolution code MESA [3].

\section{Glitch or no glitch?}

Sharp variations in the buoyancy frequency during the red giant evolution phase usually result from local changes in the chemical composition. Examples of these variations are considered here for two models of one solar mass. The position in the HR diagram of the models under consideration is shown in Figure 1 and the corresponding buoyancy frequencies in the models' inner layers are shown in Figure 2 (dashed lines).

Two spikes are visible in the buoyancy frequencies of the models under consideration. One of these spikes - at relative radii of $\sim 0.003$ (upper panel) and $\sim 0.005$ (lower panel) - results from the chemical composition variation at the hydrogen-burning shell. The shallower spike on the upper panel, at a relative radius of $\sim 0.02$, results from strong chemical gradients left behind by the retreating convective envelope which, during the first dredge up, extended to the region where the gas had previously been processed by nuclear reactions. The deeper spike visible on the lower panel, at a relative radius of $\sim 0.0008$, results from the chemical composition variation caused by a helium flash.

Whether or not the spikes in the buoyancy frequency are sufficiently sharp to produce a significant deviation of the frequencies of high radial order g-modes from their asymptotic value depends on how the characteristic width of the spikes compares with the local wavelength. A comparison of the two scales is illustrated in Figure 2, where we show in addition to the buoyancy frequency, an eigenfunction $\Psi$ (full line) related to the Lagrangian pressure perturbation, $\delta p$, for a characteristic frequency of each model (close the the model's frequency of maximum power). Here, $\Psi=\left(r^{3} / g \rho f\right)^{1 / 2} \delta p, r$ is the distance from the stellar centre, $g$ and $\rho$ are, respectively, the gravitational acceleration and density in the equilibrium model, and $f$ is a function of frequency and of the equilibrium structure (the $\mathrm{f}$ mode discriminant defined by equation (35) of [2]). From inspection of Figure 2 we see that in both models the scale in which the buoyancy frequency varies at the inner spike is much larger than the local wavelength. Hence, this spike in the buoyancy frequency is seen as a smooth variation by the wave and is well accommodated by the asymptotic analysis. In contrast, at the outermost spikes the buoyancy frequency varies on a scale comparable to or shorter than the local wavelength. We therefore may expect these features - hereafter designated by glitches - to change the oscillation frequencies from 

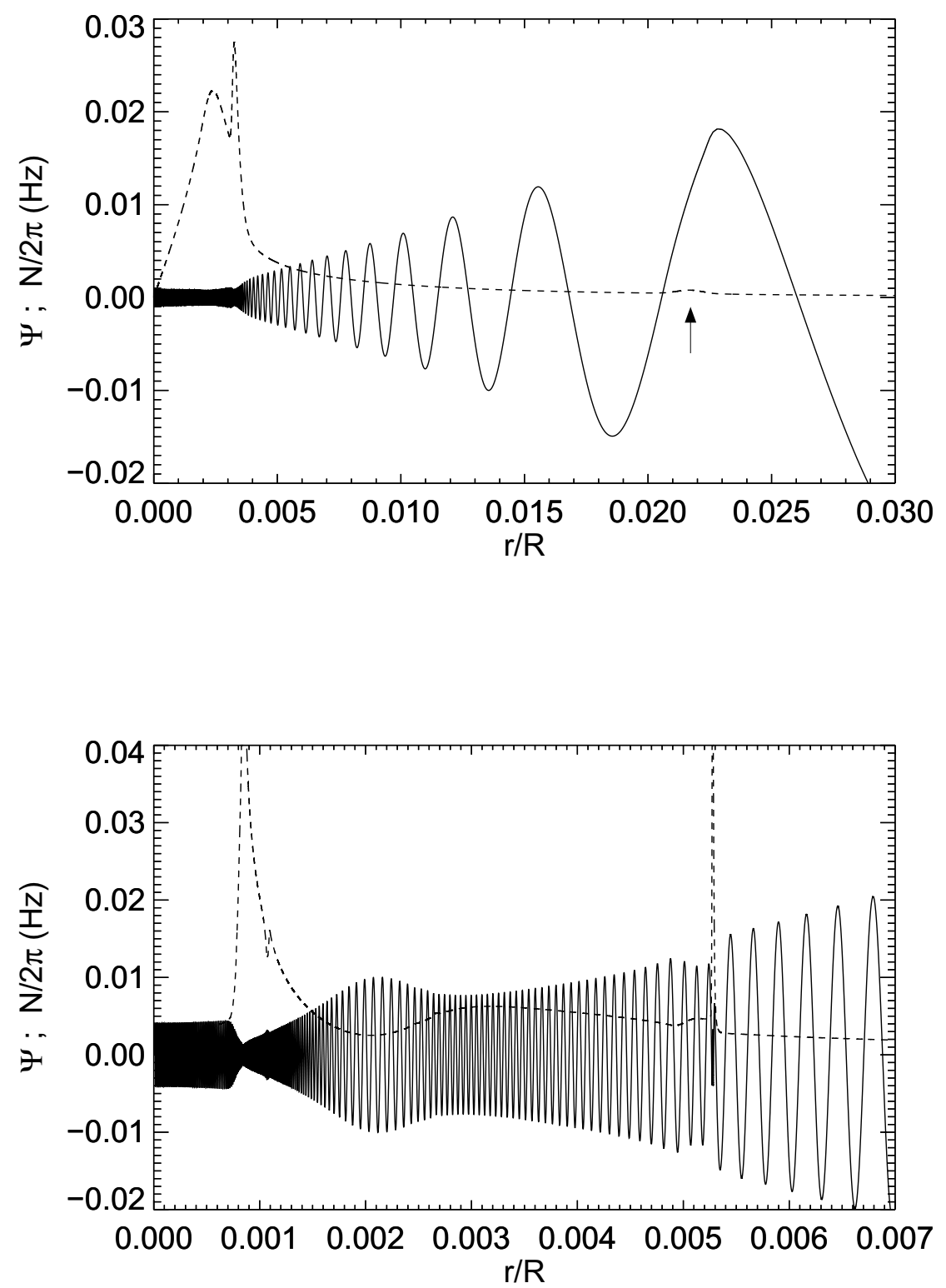

Fig. 2. Comparison between an asymptotic eigenfunction (solid line) and the buoyancy frequency (dashed line) for the red giant branch model (upper panel) and the model in between helium flashes (lower panel). The eigenfunctions have arbitrary amplitude. The arrow on the upper panel marks the position of the buoyancy frequency spike associated with the retreating of the envelope convective region (see text for details). 
their asymptotic value. In that case, the period spacings of pure g-modes may also deviate from the fixed value predicted by the asymptotic theory [4].

\subsection{Glitch effect on the period spacings}

The period spacings computed from the full numerical solutions obtained with the adiabatic pulsation code ADIPLS [1] for the two models considered in this study are shown in Figure 3 (black lines). The equally spaced dips associated with the resonant acoustic frequencies are easily identified in the red giant branch model (upper panel). In the case of the model in between helium flashes (lower panel), equally spaced deeper dips are also present, but a number of additional dips, not equally spaced in frequency, are observed between them.

To help interpret the period spacings computed from the full numerical solutions, we compare them with the period spacings computed from the numerical solutions to the pulsation equations for pure g-modes in the Cowling approximation (red lines). These are obtained solving the equation,

$$
\frac{\mathrm{d}^{2} \Psi}{\mathrm{d} r^{2}}+K^{2} \Psi=0
$$

with the wavenumber defined by

$$
K^{2}=-\frac{L^{2}}{r^{2}}\left(1-\frac{N^{2}}{\omega^{2}}\right),
$$

where $L=l(l+1), l$ is the degree of the mode, and $\omega$ is the pulsation frequency. The pulsation frequencies are derived by imposing that the solutions to equation (1) are exponentially decaying on both sides of the g-mode cavity, the latter being defined by the region inside the star where the buoyancy frequency is positive.

According to the asymptotic analysis, to first order the period spacings of pure g-modes should be constant. Nevertheless, inspection of the red lines in Figure 3 shows that the period spacings for the pure g-modes in the two models under study are modulated with frequency. This modulation is a consequence of the non-asymptotic effect introduced by the glitch in the buoyancy frequency.

Considering in more detail the full period spacings for the red giant branch model (upper panel, black line) we note that the depth of the dips associated with the resonant acoustic frequencies varies significantly. In fact, it it clear from the comparison of the black and red lines that if a dip associated with a resonant acoustic frequency coincides with a dip in the pure g-mode period spacings, its depth is smaller than if it coincides with a hump. Thus, the predominant effect of the glitch on the period spacings of this model is to introduce a frequency dependent change to the depth of the dips at the resonant acoustic frequencies.

Next we consider in more detail the full period spacings for the model in between helium flashes (lower panel, black line). The existence of closely-spaced pronounced dips in the period spacings makes it harder to identify the dips associated with the resonant acoustic frequencies in this case. Nevertheless, comparison of the black and red lines shows that the two are similar everywhere, except at the frequencies of the more pronounced dips. As the pure g-mode period spacings do not incorporate the coupling between g- and p-modes, we can safely conclude that the more pronounced dips are associated with the resonant acoustic frequencies. Likewise, we can exclude the coupling between the $\mathrm{g}$ - and p-modes as the cause of the additional dips (in between the resonant acoustic frequencies) that are seen in both the full and the g-mode period spacings for this model. As in the case of the red giant branch model, the period spacings for pure g-modes in this model would be constant, to first order, if it were not for the presence of a non-asymptotic effect caused by the glitch. In this case, the less pronounced dips seen in the period spacings derived from the full numerical solutions are caused by the outer spike in the buoyancy frequency, that is, the glitch at the hydrogen-burning shell. In addition, we note that as for the case of the red giant branch model, the glitch also affects the depth of the dips associated with the resonant acoustic frequencies. In this case the latter have a width comparable to the separation between glitch-induced dips. This similarity of scales makes it more difficult to 

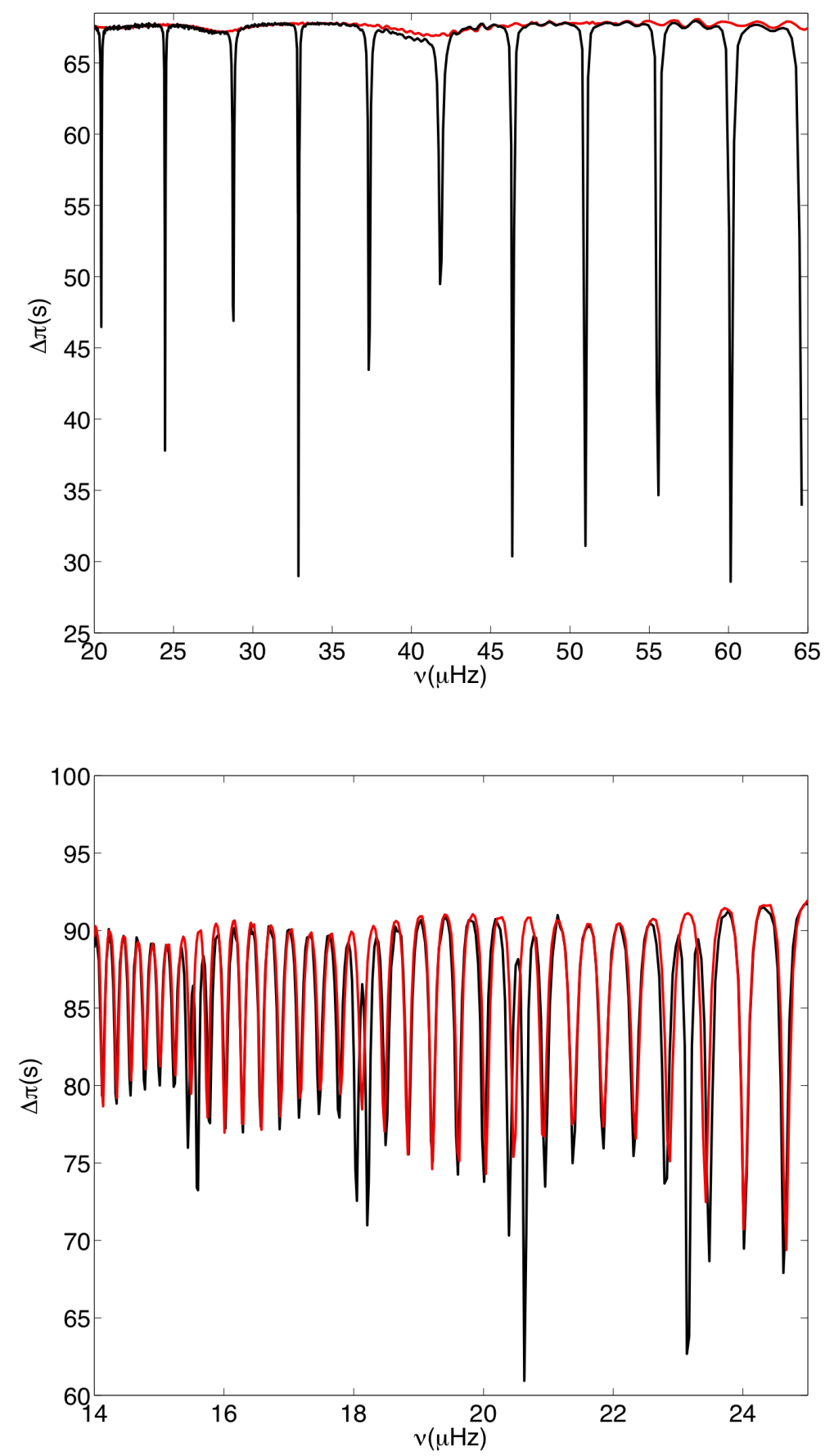

Fig. 3. Comparison of the period spacings derived from ADIPLS (black line) and from the integration of the wave equation (1) ignoring the coupling with p-modes (red line), for the red giant branch model (upper panel) and the model in between helium flashes (lower panel). 
interpret the combined effect of the glich and mode coupling. Nevertheless, a comparison between the dips at $\sim 18.1 \mu \mathrm{Hz}$ and $23.2 \mu \mathrm{Hz}$ indicates that the combined effect is the same as for the red giant branch model. While the latter dip is placed at a glitch-induced hump and, consequently, has its depth increased, the former dip is placed at a glitch-induced dip and has its central depth reduced, forming a double dip structure.

\section{Discussion}

In this work we have used two red giant models to illustrate how a glitch in the buoyancy frequency may significantly affect the period spacings of a red giant star. Comparison of the period spacings for these models with the results of a simple analytical model (Cunha et al, in preparation), further confirms that the glitches identified here are the origin of the period spacing signatures discussed above. Based on the analysis of the period spacings for a large grid of models, we are currently in the process of identifying subgroups of red-giant stars along the red giant evolution in which the signatures of glitches in the buoyancy frequency may be expected to be observed.

The authors are very grateful to the University of Sydney for providing support to this project through an IRCA Award. Funds for the project were also provided by the EC, through the FP7 project ASK (PIRSES-GA-2010269194). MSC and PPA are supported by FCT through Investigardor contracts of reference IF/00894/2012 and IF/00863/2012 and POPH/FSE (EC) by FEDER funding through the program COMPETE. Funding for the Stellar Astrophysics Centre is provided by The Danish National Research Foundation (Grant DNRF106). The research is supported by the ASTERISK project (ASTERoseismic Investigations with SONG and Kepler) funded by the European Research Council (Grant agreement no.: 267864).

\section{References}

1. Christensen-Dalsgaard, J. Ap\&SS 316, 113 (2008)

2. Gough, D. O., Astronomische Nachrichten 328, 273 (2007)

3. Paxton, B., et al., ApJS 208, 4 (2013)

4. Tassoul, M., ApJS 43, 469 (1980) 